\title{
An epidemiological study of pulmonary arterial hypertension
}

\author{
A.J. Peacock*, N.F. Murphy\#, J.J.V. McMurray\#, L. Caballero* and S. Stewart
}

ABSTRACT: All hospitalisations for pulmonary arterial hypertension (PAH) in the Scottish population were examined to determine the epidemiological features of PAH. These data were compared with expert data from the Scottish Pulmonary Vascular Unit (SPVU).

Using the linked Scottish Morbidity Record scheme, data from all adults aged 16-65 yrs admitted with PAH (idiopathic PAH, pulmonary hypertension associated with congenital heart abnormalities and pulmonary hypertension associated with connective tissue disorders) during the period 1986-2001 were identified. These data were compared with the most recent data in the SPVU database (2005).

Overall, 374 Scottish males and females aged 16-65 yrs were hospitalised with incident PAH during 1986-2001. The annual incidence of PAH was 7.1 cases per million population. On December 31, 2002, there were 165 surviving cases, giving a prevalence of PAH of 52 cases per million population. Data from the SPVU were available for 1997-2006. In 2005, the last year with a complete data set, the incidence of PAH was 7.6 cases per million population and the corresponding prevalence was 26 cases per million population.

Hospitalisation data from the Scottish Morbidity Record scheme gave higher prevalences of pulmonary arterial hypertension than data from the expert centres (Scotland and France). The hospitalisation data may overestimate the true frequency of pulmonary arterial hypertension in the population, but it is also possible that the expert centres underestimate the true frequency.

KEYWORDS: Epidemiology, incidence, prevalence, pulmonary hypertension

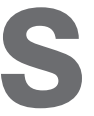
ince the late 1990s, there has been increasing interest in the causes, consequences and treatment of pulmonary arterial hypertension $(\mathrm{PAH})$, a condition of various underlying aetiologies that is defined by a mean pulmonary arterial pressure of $>25 \mathrm{mmHg}$ at rest or $>30 \mathrm{mmHg}$ on exercise in the absence of significant lung or left heart disease. Until the introduction by the World Health Organization of a formal classification scheme for coding any form of pulmonary hypertension $(\mathrm{PH}$; recently updated by an expert consensus panel in June 2003), and formal recommendations for its management $[1,2]$, it was difficult for clinicians to determine the scope of the problem and apply definitive treatment.

With the exception of a recent French report [3], there are few data describing the incidence and prevalence of the most common forms of $\mathrm{PAH}$, including its idiopathic form (IPAH) and those relating to connective tissues disorders (CTD$\mathrm{PAH})$ and congenital heart disease (CHD-PAH).

For editorial comments see page 1.
The recently published French National Registry data suggest an overall prevalence of PAH of 15 cases per million population, but, although $\mathrm{PH}$ is considered to have a homogenous distribution, there was considerable variation across France (5-25 cases per million population).

\section{STUDY AIMS}

In this context, the aim of the present study was to examine the epidemiological features of PAH within a whole population over a prolonged period from two perspectives, inexpert and expert, a national hospitalisation registry and the specialist centre for $\mathrm{PAH}$ management in Scotland.

\section{METHODS}

\section{National hospitalisation registry data for} Scotland

In the present study, records from the Scottish Morbidity Record (SMR) scheme [4] were specifically examined. This validated data set captures and records all hospitalisations in Scotland on an individual basis [5], and has previously been used to describe trends in cardiovascular morbidity and mortality within the whole Scottish
AFFILIATIONS

*Scottish Pulmonary Vascular Unit, Western Infirmary, and

\# Dept of Cardiology, University of Glasgow, Glasgow, UK.

- Preventative Cardiology, Baker Heart Research Institute, Melbourne, Australia.

CORRESPONDENCE

A.J. Peacock

Scottish Pulmonary Vascular Unit

Western Infirmary

Glasgow

G61 2SW

UK

Fax: 441412116334

E-mail: apeacock@udcf.gla.ac.uk

Received:

July 122006

Accepted after revision:

February 262007

SUPPORT STATEMENT

S. Stewart is supported by the National Heart Foundation (Melbourne, Australia) and National Health and Medical Research Council of Australia (Canberra, Australia)

\section{STATEMENT OF INTEREST}

Statements of interest for all authors of this manuscript can be found at www.erj.ersjournals.com/misc/ statements.shtml

European Respiratory Journa Print ISSN 0903-1936

Online ISSN 1399-3003 
population (total 5 million) [6, 7]. As described more extensively in previous reports $[6,7]$, the Information and Statistics Division of the National Health Service in Scotland collects and collates data regarding all hospital discharges via the SMR scheme [4]. Data from patient case records are used to code up to six diagnoses at the time of each hospital discharge occurring in that country. Currently, the tenth revision of the World Health Organization International Statistical Classification of Diseases and Related Health Problems (ICD10 ) is used to code discharges [8], with the ninth revision (ICD9) being used until 1996 [9].

The term discharge includes both live and fatal discharges. These data are linked to information held by the General Register Office for Scotland relating to all deaths, and records are maintained using a unique identifier for each patient. Consequently, any readmission or death (and its cause) can be identified for each individual who is admitted to hospital in Scotland. Each hospital record provides information concerning the individual's age, sex, date of admission and usual postcode of residence. They also provide detailed information on duration of stay, type of hospital unit and destination (e.g. home or institutional care) following a live discharge.

\section{Diagnostic codes}

Using SMR data, all individuals aged 16-65 yrs who were discharged for the first time from a Scottish hospital during the 16-yr period, 1986-2001, with a discharge diagnosis of primary PH/PAH were identified (ICD-9 code 416.0; ICD-10 code I27.0).

\section{Data verification}

It is important to note that the coding instructions indicate that the target code for PAH be used to denote arterial as opposed to venous $\mathrm{PH}$, and auditing of SMR coding confirmed a high degree of accuracy in coding practices overall [5]. However, close analysis of the coding of all forms of $\mathrm{PH}$ requires careful interpretation. Therefore, stringent case definitions were applied to exclude cases in which $\mathrm{PH}$ was probably miscoded as PAH.

\section{Case definitions}

In order to delineate the most common forms of PAH and other forms of $\mathrm{PH}$ (see below) captured by these data, the combination of concurrently recorded diagnoses and coding data from any prior hospitalisations was used. Using these data, a series of filters, with a hierarchal order of priority, were applied to categorise each patient according to the latest classification system published in 2004 [2]. In order to further increase the probability that a diagnosis of PAH was based on a series of clinical investigations with determination of concurrent disease states, the present analyses were confined to adults aged $\leqslant 65$ yrs.

\section{Pulmonany arterial hypertension}

First, all patients in whom an isolated coding of PAH was recorded in the absence of any explanatory concurrent diagnoses from prior hospitalisations were identified and designated as having IPAH. All individuals with a past or concurrent diagnosis of congenital systemic-to-pulmonary shunts (e.g. ICD-9 code 747.3) were designated, regardless of other potentially listed causes, as having CHD-PAH. Similarly, patients with evidence of $\mathrm{PAH}$ related to systemic connective tissue disorders (e.g. ICD-10 codes M30-M36) were identified and designated as having CTD-PAH.

Other potentially relevant cases (e.g. PAH related to HIV infection/treatment) occurred in $<10$ patients over the $16-y r$ period and were, therefore, not specifically identified.

\section{Pulmonary hypertension}

The remaining patients were categorised in the following order and manner. Any patient with a history of left-sided heart failure or valvular disease (e.g. ICD-9 code 428.1: left-sided heart failure), regardless of other potential causes, was designated as having $\mathrm{PH}$ with left heart disease. Similarly, any patient with a history of chronic respiratory disease (e.g. ICD-9 codes 460-5190) or hypoxia was designated as having $\mathrm{PH}$ with lung disease and/or hypoxaemia, or respiratory $\mathrm{PH}$. Patients with $\mathrm{PH}$ with concurrent thomboembolic disease (e.g. ICD-9 code 415.1: pulmonary embolism) were designated as having thromboembolic PH. Data specific to these cases were excluded from the present article.

\section{Study cohort}

During the 16-yr period, 1986-2001, a total of 4,794 patients were discharged from Scottish hospitals with a first-ever diagnosis of primary $\mathrm{PH} / \mathrm{PAH}$. A total of 2,198 (46\%) of these patients were in the first or second diagnostic position for coding purposes and aged $\leqslant 65$ yrs. Using the coding algorithms described above, a total of 374 cases, male and female and aged 16-65 yrs, were identified to be associated with a probable diagnosis of PAH (i.e. consistent with the current classification system). Of the study cohort, $\sim 80 \%$ were discharged from hospitals with specialist respiratory and cardiology services (60\% from tertiary affiliated hospitals).

\section{National unit data: the Scottish Pulmonary Vascular Unit}

The Scottish Pulmonary Vascular Unit (SPVU) is responsible for the diagnosis and treatment of all cases of PAH in Scotland (population $\sim 5$ million). The unit has been collecting data regarding referred cases since 1997, and there are relatively complete data sets for the last 4 yrs (i.e. 2002-2005). All cases of $\mathrm{PAH}$ are investigated using a standard diagnostic protocol that includes right heart catheterisation. $\mathrm{PAH}$ is diagnosed only if mean pulmonary arterial pressure is $>25 \mathrm{mmHg}$ at rest, with a pulmonary artery wedge pressure of $<15 \mathrm{mmHg}$. Patients with severe abnormalities in pulmonary function (forced expiratory volume in one second of $<60 \%$ ) are excluded from analysis.

\section{Data analysis}

Hospitalisation data

At the time of the present analysis, the linked SMR database permitted reliable analysis of survival data until December 31, 2002. All surviving patients were censored at this time-point in order to provide a follow-up of 1-16 yrs for every patient. Incidence rates were calculated from official Scottish population census data on an age- and sex-specific basis. The point prevalence of PAH on December 31, 2002 was calculated from the number of incident cases who were still alive at this point (i.e. it does not take into consideration individuals who have not been hospitalised for PAH or who were admitted to a Scottish hospital prior to 1986). Surviving cases were related to the size of the total population in the appropriate age groups (3.2 million). 


\section{SPVU data}

Data from patients with $\mathrm{PH}$ has been recorded in the SPVU database since 1997. During the period 1997-2005, there has been a progressive increase in both the prevalence and incidence of $\mathrm{PAH}$, suggesting that an increasing number of patients are being discovered rather than there being a genuine change in the frequency of cases in this population. Therefore, the incidence data from 2005 (the last year with a complete data set) were used in the present study. The prevalence data describe the point prevalence of $\mathrm{PAH}$ and its three subtypes amongst survivors on December 31, 2005 relative to the total Scottish population (5 million).

\section{RESULTS}

\section{National hospitalisation cohort: SMR data}

\section{Profile}

During the 16-yr period, 1986-2001, a total of 374 males and females aged 16-65 yrs who were discharged from a Scottish hospital for the first time with a diagnosis of IPAH, CTD-PAH or CHD-PAH were identified. Table 1 shows the distribution of incident cases according to age, sex and diagnostic classification. Overall, females outnumbered males by almost 2:1 and were significantly older ( $\mathrm{p}<0.001$ for all comparisons).

\section{Population incidence}

In Scottish males aged 16-65 yrs, the overall annual incidence of IPAH during the 16-yr study period was 2.5 cases per million population ( 2.7 and 3.0 cases per million population in 1986 and 2001, respectively). The equivalent annual data for CTD-PAH and CHD-PAH were 0.7 (0.0 and 0.6) and 1.1 (1.1 and 1.2) cases per million population, respectively. The annual incidence of all forms of $\mathrm{PAH}$, including IPAH, remained essentially stable in males during the study period.

In Scottish females, incidences of PAH were higher ( 1.5-3:1) than in Scottish males. The overall annual incidence of IPAH during the 16-yr study period was 4.0 cases per million population (4.7 and 4.1 cases per million population in 1986 and 2001, respectively). The equivalent annual data for CTDPAH and CHD-PAH were 3.4 (1.8 and 5.9) and 2.2 (0.5 and 2.4) cases per million population, respectively. The annual

\begin{tabular}{lccccc} 
TABLE 1 & $\begin{array}{l}\text { Demographic profile, including type of } \\
\text { pulmonary arterial hypertension, of the national } \\
\text { hospitalisation cohort SMR data\# }\end{array}$ \\
\cline { 2 - 3 } & \multicolumn{3}{c}{ Males } & & \multicolumn{2}{c}{ Females } \\
\cline { 2 - 3 } \cline { 5 - 6 } & Subjects & Age & & Subjects & Age \\
& $\mathbf{n}$ & yrs & & $\mathbf{n}$ & yrs \\
\hline Idiopathic & 66 & $47 \pm 12$ & & 109 & $49 \pm 11$ \\
Connective & 19 & $51 \pm 10$ & & 92 & $52 \pm 11$ \\
Congenital & 28 & $44 \pm 17$ & 60 & $44 \pm 15$ \\
Total & 113 & $50 \pm 13$ & 261 & $52 \pm 12$ \\
\hline
\end{tabular}

Data are presented as mean $\pm S D$, unless otherwise stated. SMR: Scottish Morbidity Record. \#: Scottish males and females aged 16-65 yrs who were discharged from a Scottish hospital for the first time during the period 1986-2001. incidence of all forms of $\mathrm{PAH}$, including IPAH, remained essentially stable in females during the study period. The combined male and female incidences of PAH and its subtypes are shown in table 2 and figure 1.

\section{Point prevalence of PAH}

A total of 58 males and 107 females discharged from hospital with a diagnosis of PAH during the previous 16 yrs survived until December 31, 2002. The median survival in those individuals with IPAH was 3.8 yrs and 5.6 yrs in females and males, respectively. The equivalent data for $\mathrm{CHD}-\mathrm{PAH}$ and CTD-PAH were 5.9 and 4.5 yrs in males and 4.9 and $2.6 \mathrm{yrs}$ in females, respectively. The overall population prevalence in the adult (16-65 yrs) population of Scotland ( $\sim 3.2$ million) of all three forms of PAH at this time-point was 52 cases per million population (table 2; fig. 2 ).

\section{SPVU data set}

In 2005, the combined annual incidences (for males and females) of IPAH, CTD-PH and CHD-PH in the adult population were 2.6, 2.8 and 2.2 cases per million population, respectively. These contributed to a total annual incidence recorded at the Scottish expert centre of 7.6 cases per million population (table 2; fig. 1)

On December 31, 2005 the prevalence of patients living with IPAH, CTD-PH and CHD-PH were 9, 10 and 7 cases per million population, respectively, contributing to a total prevalence of 26 cases per million population recorded at the SVPU.

The data from each of the three sources (Scottish hospitalisation records, the SPVU and the French national registry) have been combined in figures 1 and 2 (incidence and prevalence, respectively).

\section{DISCUSSION}

The present unique study examined the epidemiological profile of $\mathrm{PAH}$ from two perspectives, principally from hospitalisation data, where diagnosis was usually inexpert,

\begin{tabular}{lllll} 
TABLE 2 & $\begin{array}{l}\text { Prevalence and incidence of pulmonary arterial } \\
\text { hypertension and its subtypes from three } \\
\text { sources }\end{array}$ \\
& Total & IPH & CTD-PH & CHD-PH \\
\hline & & & & \\
\hline $\begin{array}{l}\text { Prevalence } \\
\text { French }\end{array}$ & 15 & 6.5 & 2.3 & 1.7 \\
SMR & 52 & 25 & 15 & 12 \\
$\quad$ SPVU & 26 & 9 & 10 & 7 \\
Incidence & & & & \\
French & 2.4 & 1.0 & 0.4 & 0.3 \\
SMR & 7.1 & 3.3 & 2.1 & 1.7 \\
SPVU & 7.6 & 2.6 & 2.8 & 2.2 \\
\hline
\end{tabular}

Data are presented as cases per million population and annual cases per million population for prevalence and incidence, respectively. IPH: idiopathic pulmonary hypertension (PH); CTD-PH: connective tissue disease-associated PH; CHD-PH: congenital heart disease-associated PH; SMR: Scottish Morbidity Record; SPVU: Scottish Pulmonary Vascular Unit. ${ }^{*}$ : SMR, SPVU database and the French national registry. 


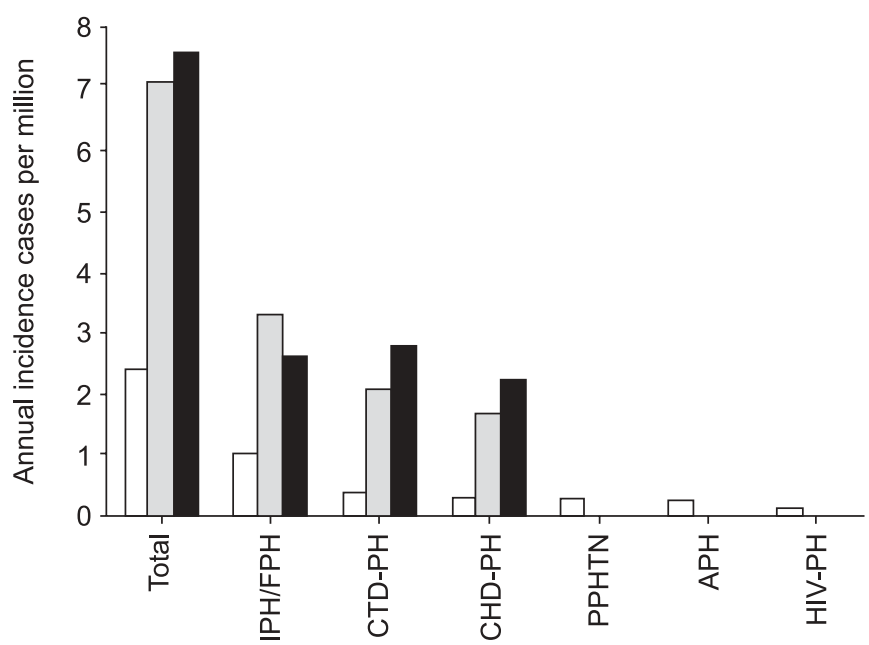

FIGURE 1. Incidence of pulmonary arterial hypertension in Scotland and France from three sources. $\square$ : French national registry; $\square$ : Scottish hospitalisation records (Scottish Morbidity Record); ם: Scottish Pulmonary Vascular Unit database. IPH: idiopathic pulmonary hypertension (PH); FPH: familial $\mathrm{PH}$; CTD$\mathrm{PH}$ : connective tissue disease-associated $\mathrm{PH}$; $\mathrm{CHD}-\mathrm{PH}$ : congenital heart diseaseassociated $\mathrm{PH}$; PPHTN: portopulmonary hypertension; $\mathrm{APH}$ : $\mathrm{PH}$ associated with other diseases or things, in this case anorexigen; HIV-PH: HIV-associated $\mathrm{PH}$

but also from data collected at the Scottish expert centre, where the diagnostic criteria were similar to those of the French national registry.

Prior to the French study [3], there were studies containing epidemiological components. The USA National Institutes of Health (NIH) registry was started in 1981, and results were published in 1987 [10]. There were 187 patients with primary PH (now called IPH), with a mean age of 36 yrs and a female/ male ratio of 1.7:1. From this study, it was concluded that the annual incidence of primary $\mathrm{PH}$ was 1-2 cases per million population. In 1991, D'ALONZO et al. [11] published survival data for these patients. The NIH registry did not attempt to describe or make any further estimates of incidence or prevalence. In 1996, a study was published that examined the relationship of primary $\mathrm{PH}$ (now IPH) to appetite suppressant use [12]. They recruited patients diagnosed with primary PH between September 1, 1992 and September 30, 1994 from 220 centres in the UK, France, Belgium and the Netherlands. A total of 135 patients were recruited, although, in addition, 26 were dead or too sick to be interviewed. Of the 135,23 were thought not to have primary $\mathrm{PH}$ and 17 could not be examined, leaving 95 cases with definite $(n=80)$ or probable $(\mathrm{n}=15)$ primary $\mathrm{PH}$. This study was largely about the relative risk of appetite suppressants, but the annual incidence of IPH was calculated in Belgium, where it was found to be 1.7 cases per million population (95\% confidence interval 1.0-2.4). In 2005, the Centers for Disease Control and Prevention published a surveillance study of $\mathrm{PH}$ covering the period 19802002 [13]. This summarised mortality data from the National Vital Statistics System and hospital data from the National Hospital Discharge Survey, but no attempt was made to calculate the incidence or prevalence of the disease in the USA.

The French national registry collected the records of patients from 17 centres in France during the period October 2002 to

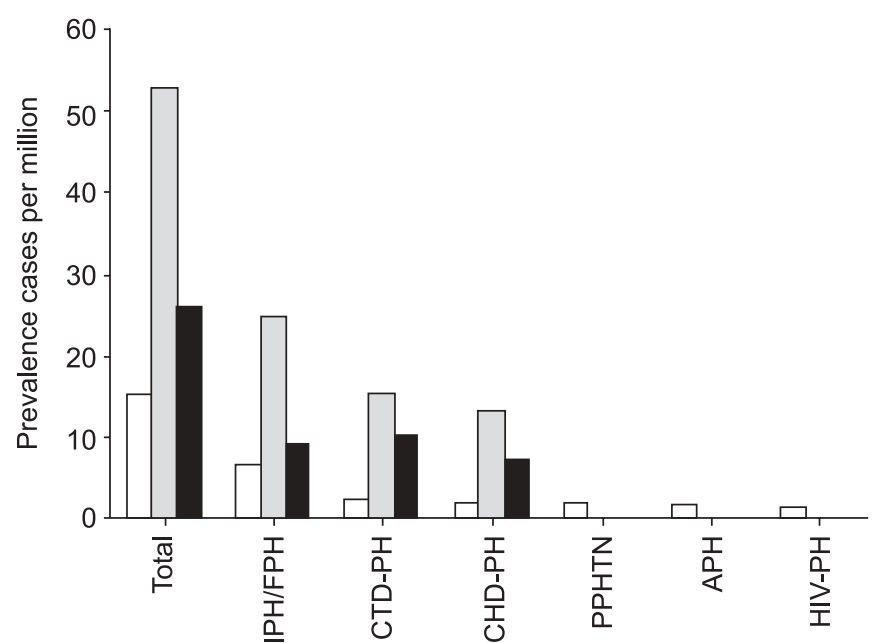

FIGURE 2. Prevalence of pulmonary arterial hypertension in Scotland and France from three sources. $\square$ : French national registry; $\square$ : Scottish hospitalisation records (Scottish Morbidity Record); $\mathbf{\square}$ : Scottish Pulmonary Vascular Unit database. IPH: idiopathic pulmonary hypertension (PH); $\mathrm{FPH}$ : familial $\mathrm{PH}$; CTD$\mathrm{PH}$ : connective tissue disease-associated $\mathrm{PH}$; CHD-PH: congenital heart diseaseassociated PH; PPHTN: portopulmonary hypertension; APAH: $\mathrm{PH}$ associated with other diseases or things, in this case anorexigen; HIV-PH: HIV-associated PH.

October 2003 [3]. All patients required cardiac catheterisation. $\mathrm{PAH}$ was only diagnosed if strict cardiac catheter criteria were followed, i.e. a mean pulmonary arterial pressure of $>25 \mathrm{mmHg}$. It also excluded significant lung disease. A total of 674 patients were entered into the database. The calculated total prevalence and annual incidence of PAH were 15 and 2.4 cases per million population, respectively. Interestingly, there was variation in the prevalence across France of 525 cases per million population, suggesting to the authors that their overall prevalence of 15 cases per million population was probably an underestimate. Additionally, $25 \%$ of their cases were aged $>60$ yrs, which was quite different from the NIH registry but similar to the data held on the Scottish national database.

Although PAH has previously been accepted as a rare and deadly disease state, no studies have examined its epidemiology beyond the experiences and records of specialist centres and national registries. By their very nature, these are more likely to describe a younger, more select group of patients. In previous studies, therefore, the annual incidences of IPAH and CTD-PAH have been reported to be $1-2$ cases per million population. There was also a preponderance of relatively young females. Using whole-population morbidity data from Scotland, $\sim 400$ incident cases of PAH were identified in the present study in males and females aged 16-65 yrs during the period 1986-2001. Although it was confirmed that PAH is predominantly a female condition, even with an age restriction to increase the accuracy of diagnostic codes, both the male and female patients in the present study were older than their counterparts in previous reports. Moreover, the underlying annual incidence of IPAH in the present study, even taking into account the fact that the focus was on patients aged $\leqslant 65$ yrs, was higher than that in previous reports, being 2.5 and 4.0 cases per million population in males and females, 
respectively. Although the annual incidences of CTD-PAH and $\mathrm{CHD}-\mathrm{PAH}$ in males were consistent with previous reports $(\sim 1$ case per million population), they were approximately two- or three-fold higher (2.2 and 3.4 cases per million population) in females. During the prolonged follow-up period, these incidences were essentially stable over time.

Using a combination of prevalence and survival data, it was possible to determine the point prevalence of $\mathrm{PAH}$ and other forms of PH in the Scottish population at the end of 2002 in the present study. Although these data do not take into account patients who survived hospitalisation prior to 1986, or individuals not diagnosed or treated via the hospital system in Scotland, they do give some indication of the current burden of PAH in a developed country. As such, in a population of $\sim 3.2$ million aged 16-65 yrs, it was found that $\sim 100$ adults were still alive at the end of the study period following a hospital admission for IPAH, and slightly smaller numbers for CTD-PAH and CHD-PAH. Given the combination of stable incidence rates but more prolonged survival, the population prevalence of this condition is likely to increase rather than decrease well into the 2010s. Moreover, with expanding medical [14, 15] and surgical options [16, 17] and increased awareness of $\mathrm{PAH}$ in particular, it is possible that specialist treatment centres will start to treat a greater number of older patients with this condition.

The SPVU, founded in 1997, is the national referral centre for $\mathrm{PH}$ in Scotland. There has been a steady increase in referral to the unit, but this stabilised during 2004 and 2005. The unit receives referrals of cases of IPH and CTD-PH and some, but not all, cases of CHD-PH, similar to the French registries. Diagnostic criteria are very similar to those used by the French centres. Of the patients, $\sim 30 \%$ are aged $>65$ yrs. The unit database suggests a prevalence of $\mathrm{PAH}$ of 26 cases per million population and an annual incidence of PAH of 7.6 cases per million population.

The present study of hospital discharge records has a number of obvious limitations that require comment. As noted in the Methods section, it is a retrospective population cohort study that relies upon the systematic coding of hospital admissions in the absence of definitive guidelines for the diagnosis of $\mathrm{PAH}$ and other clinically important forms of $\mathrm{PH}$. In order to address this issue, a systematic and conservative screening process was applied to all incident admissions associated with a diagnosis of PAH, using the latest diagnostic categories [1, 2], and analyses confined to individuals aged 16-65 yrs, in whom more accurate diagnoses were likely to have been made [5]. If individuals aged $\geqslant 65$ yrs (i.e. the entire adult population) had been included in the present analysis, the reported incidence rates of IPAH and CTD-PAH, in 2001, would have been 4 and 2 cases per million for males and 8 and 7 cases per million for females, respectively. Significantly, no clinical data (most notably pulmonary arterial pressures) were available and it was, therefore, not possible to verify that each and every case had been accurately diagnosed.

The present study of hospitalisation records, therefore, represents a survey of inexpert diagnoses that would have been made using a number of investigatory techniques, unlike the French study, which restricted the diagnosis to those patients who had been studied by invasive cardiac catheterisation. The appropriately anonymous nature of the SMR scheme does not permit verification of data in individual cases. Comparisons with other data sets and anecdotal reports (particularly the preponderance of females, survival rates in different subtypes of PAH and changes in survival rates), however, suggest that these data are of clinical validity and provide a reasonably accurate, and even conservative, study of the epidemiological features of PAH within a whole population. The most recently published study from an expert centre, the French study [3], also has limitations; in particular, only patients who had received a catheter diagnosis at an expert centre were included, and it is notable that there was wide variation in the prevalence of PAH in the French data set, with higher prevalences seen in the Paris region (the region with the best-known and largest $\mathrm{PH}$ centre). Perhaps the two approaches provide an estimate of the minimum (French study) and near-maximum (Scottish hospitalisation study) incidences and prevalences of the various subtypes of PAH. Interestingly, although the absolute incidences and prevalences of $\mathrm{PH}$ differ considerably between the Scottish and French data sets, the proportion of subtypes in the two populations are similar, suggesting that the two approaches are comparable (figs 1 and 2). Furthermore, the data from the expert centre in Scotland, the SPVU, shows similar incidences to those obtained from the hospitalisation study, although the prevalences are lower and closer to those of the French registry. It must be remembered, however, that the data from the SPVU are from a later cohort than the hospitalisation study and include all age groups rather than being restricted to those aged 16-65 yrs.

In conclusion, this unique population cohort study suggests that previous reports, from specialist centres of pulmonary arterial hypertension management, have underestimated the true incidence and prevalence of this condition, but confirmation of this speculation requires analysis of detailed registries from each country, which are in the process of being collected.

\section{REFERENCES}

1 Gibbs JSR, Higenbottam TW, for the British Cardiac Society Guidelines and Medical Practice Committee. Recommendations on the management of pulmonary hypertension in clinical practice. Heart 2001; 86: Suppl. I, i1-i13.

2 Simmoneau G, Nazzareno G, Rubin LJ, et al. Clinical classification of pulmonary hypertension. J Am Coll Cardiol 2004; 43: Suppl. 12, 5S-12S.

3 Humbert M, Sitbon O, Chaouat A, et al. Pulmonary arterial hypertension in France: results from a national registry. Am J Respir Crit Care Med 2006; 173: 1023-1030.

4 Kendrick S, Clarke J. The Scottish record linkage system. Health Bull 1993; 51: 72-79.

5 Harley K, Jones C. Quality of Scottish Morbidity Record (SMR) data. Health Bull 1996; 54: 410-417.

6 MacIntyre K, Capewell S, Stewart S, et al. Evidence of improving prognosis in heart failure. Trends in case fatality in 66547 patients hospitalized between 1986 and 1995. Circulation 2000; 102: 1126-1131.

7 Capewell S, MacIntyre K, Stewart S, et al. Age, sex and social trends in out-of-hospital cardiac deaths in Scotland 
1986-95: a retrospective cohort study. Lancet 2001; 358: 1213-1217.

8 World Health Organization. Manual of the International Statistical Classification of Diseases, Injuries and Causes of Death. 10th revision. Geneva, World Health Organization, 1996.

9 World Health Organization. Manual of the International Statistical Classification of Diseases, Injuries and Causes of Death. 9th revision. Geneva, World Health Organization, 1977.

10 Rich S, Dantzker DR, Ayres SM, et al. Primary pulmonary hypertension. A national prospective study. Ann Intern Med 1987; 206: 216-223.

11 D'Alonzo GE, Barst RJ, Ayers SM, et al. Survival in patients with primary pulmonary hypertension. Results from a national prospective registry. Ann Intern Med 1991; 115: 343-349.

12 Abenhaim L, Moride Y, Brenot F, et al. Appetite suppressant drugs and the risk of primary pulmonary hypertension. International Primary Pulmonary Hypertension Study Group. N Engl J Med 1996; 335: 609-616.
13 Pulmonary Hypertension Surveillance - United States, 2002. CDC Surveillance Summaries, November 11, 2005; 54: SSO5, 1-28.

14 McLaughlin VV, Presberg KW, Doyle RL, et al. Prognosis of pulmonary arterial hypertension: ACCP evidence-based clinical practice guidelines. Chest 2004; 126: Suppl. 1, 78S-92S.

15 Galie N, Seeger W, Naeije R, Simmoneau G, Rubin LJ. Comparative analysis of clinical trials and evidence-based treatment algorithm in pulmonary arterial hypertension. J Am Coll Cardiol 2004; 43: Suppl. 12, S76-S88.

16 Dartevelle P, Fadel E, Mussot S, et al. Chronic thromboembolic pulmonary hypertension. Eur Respir J 2004; 23: 637-648.

17 Keck BM, Bennett LE, Fiol BS, Daily OP, Novick RJ, Hosenpud JD. Worldwide thoracic organ transplantation: a report from UNSO/ISHLT International Registry for Thoracic Organ Transplantation. Clin Transpl 1998; 1998: 39-52. 